Original

\title{
Thermographic assessment of facial temperature in patients undergoing orthognathic surgery
}

\author{
Toshiya Endo ${ }^{1)}$, Akira Komatsuzaki' ${ }^{2)}$, Yukio Miyagawa ${ }^{3)}$, Takeshi Kamoda ${ }^{2}$, \\ Sho Goto $^{1)}$, Katsunori Koide ${ }^{1)}$, and Masutaka Mizutani ${ }^{4}$ \\ 1)Department of Orthodontics and Dentofacial Orthopedics, Graduate School of Life Dentistry at Niigata, \\ The Nippon Dental University, Niigata, Japan \\ 2)Department of Preventive and Community Dentistry, The Nippon Dental University School \\ of Life Dentistry at Niigata, Niigata, Japan \\ 3)Department of Dental Materials Science, The Nippon Dental University School of Life Dentistry at Niigata, \\ Niigata, Japan \\ 4)Oral and Maxillofacial Surgery, The Nippon Dental University Niigata Hospital, Niigata, Japan
}

(Received June 6, 2018; Accepted October 2, 2018)

\begin{abstract}
This study was conducted to assess the usefulness of thermography for quantifying facial temperature before and after orthognathic surgery and intermaxillary fixation, and the effects of these orthognathic procedures on facial temperature. Facial thermograms of 10 patients who underwent bilateral sagittal split ramus osteotomy (SSRO, one-jaw group) and another 10 patients who underwent Le Fort I osteotomy and bilateral SSRO (two-jaw group) were taken 1 day before orthognathic surgery (T1) and at release of intermaxillary fixation 7 days later (T2). Two thermograms taken $30 \mathrm{~s}$ (TG1) and $3 \mathrm{~min}$ (TG2) after the start of recording at $\mathrm{T} 1$ and $\mathrm{T} 2$ were used. A square $(26 \times 26$ pixels $)$ was marked on each thermogram and the mean facial temperature for each square was measured. Three-way analysis of variance was used for statistical comparisons. Facial temperatures were significantly higher at $\mathrm{T} 2$ than at T1 on TG1 and TG2, and were significantly higher on TG2 than on TG1 at T1 and T2. The two-jaw group
\end{abstract}

Correspondence to Dr. Toshiya Endo, Department of Orthodontics and Dentofacial Orthopedics, Graduate School of Life Dentistry at Niigata, The Nippon Dental University, 1-8 Hamaura-cho, Chuo-ku, Niigata 951-8580, Japan

Fax: +81-25-265-5819 E-mail: endoto@ngt.ndu.ac.jp

Color figures can be viewed in the online issue at J-STAGE. doi.org/10.2334/josnusd.18-0194

DN/JST.JSTAGE/josnusd/18-0194 had a significantly higher facial temperature than the one-jaw group. Thermography was useful for quantitative assessment of facial temperature in patients undergoing orthognathic surgery. Changes in facial temperature were due predominantly to inflammation after surgery, rather than to sarcopenia.

Keywords: thermography; facial temperature; orthognathic surgery; intermaxillary fixation.

\section{Introduction}

Orthognathic surgery is now performed worldwide as the mainstay treatment for jaw deformities, allowing improvement of not only facial aesthetics, malocclusion, oral health and function, but also mental well-being (1). Intermaxillary fixation has been used for achieving proper occlusion after orthognathic surgery (2). Weight loss, and decreased jaw and pulmonary function have been noted as the main side effects of intermaxillary fixation (3). The reported biologic alterations resulting from orthognathic surgery and intermaxillary fixation include muscle atrophy, denervation, alteration of fiber types, myofibrosis, decreased muscle mass, and morphologic alterations of the condyle $(3,4)$. These conditions lead to sarcopenia, which is a loss of skeletal muscle mass, function or strength (5).

Thermography produces thermal images representing the spatial temperature distribution on the outer surface of 
human skin by monitoring the emitted infrared radiation (6). In the field of dentistry, thermography has been used to evaluate the temperature changes in bones during dental implant site preparation (7), in enamel surfaces during tooth bleaching (8), and in cross-sectioned teeth during $\mathrm{CO}_{2}$ laser debonding of ceramic brackets (9). Moreover, this technique has been employed as a clinically relevant tool for assessing chronic orofacial pain disorders (10), temporomandibular joint disorders $(6,11)$, and inferior alveolar nerve injury (12). Previous studies have confirmed that thermography is very useful for objective quantification of inflammation after surgical removal of mandibular third molars (13-15) and assessing the effects of agents such as analgesics and anti-inflammatory drugs $(13,14)$. Another study has reported that thermography is not sufficiently sensitive for investigating the inflammatory response to mandibular third molar extraction but may be useful for assessing inflammation after extensive surgical procedures such as orthognathic surgery (16). Currently, it appears that no previous study has employed thermography to assess the effects of orthognathic surgery and intermaxillary fixation on facial temperature.

It would perhaps be expected that facial temperature would decrease with time after orthognathic surgery, and that such patients would leave hospital once their facial temperature has normalized. For such patients, it is critical but difficult to decide the timing of discharge from hospital (17), and therefore longitudinal thermography could be potentially useful in this setting.

The present study is the first to evaluate facial temperatures before and after orthognathic surgery and intermaxillary fixation using thermography. The purpose of the study was to assess 1) the usefulness of thermography for quantifying facial temperature before and after orthognathic surgery and intermaxillary fixation, and 2) the effects of these orthognathic procedures on facial temperature. The null hypothesis tested was that orthognathic surgery and intermaxillary fixation would have no effect on facial temperature.

\section{Materials and Methods}

The protocol of this study was approved by the Research Ethics Committee of The Nippon Dental University (ECNG-R-320). Informed consent was obtained from all participants, who had been given written and oral information about this project. Sample size was determined based on a prior power analysis using $\mathrm{G}^{*}$ power software (version 3.1.9.2, Heinrich, Heine University, Dusseldorf, Germany) for three-way repeated-measures analysis of variance (ANOVA) at an effect size of 0.4 (Cohen's large effect size), an alpha error probability of 0.05 , a power of
0.8, two groups, two measurements, a correlation among repeated measures of 0.5 and non-sphericity correction of 1 (18). As power analysis showed that 16 participants were required for the within-between interaction, the sample size in each group was set at 10 participants.

\section{Participants}

Twenty patients who had undergone surgery for jaw deformities were selected retrospectively from the files of the orthodontic clinic at The Nippon Dental University Niigata Hospital. The patients were divided into two groups according to the types of orthognathic surgery they had received: a one-jaw group of 10 patients (one male and nine female) who had undergone bilateral sagittal split-ramus osteotomy (SSRO) for correction of skeletal Class III deformity characterized by mandibular prognathism, and a two-jaw group of 10 patients (six male and four female), who had been diagnosed as having skeletal Class III deformity characterized by mandibular prognathism and maxillary deficiency and who had undergone both Le Fort I osteotomy and bilateral SSRO. After orthognathic surgery, all patients in the one- and two-jaw groups underwent intermaxillary fixation for 7 days. To achieve intermaxillary fixation, surgical hooks attached to orthodontic wires were anchored with chaintype elastics on the first postoperative day, and then with ligature wires after the second postoperative day. The mean degree of mandibular set-back was $5.2 \mathrm{~mm}(\mathrm{SD}=$ $3.2 \mathrm{~mm})$ in the one-jaw group and $7.8 \mathrm{~mm}(\mathrm{SD}=2.9 \mathrm{~mm})$ in the two-jaw group, and the mean degree of maxillar set-forward was $3.5 \mathrm{~mm}(\mathrm{SD}=1.3 \mathrm{~mm})$ in the two-jaw group. The mean age at jaw surgery was 31.5 years $(\mathrm{SD}=$ 11.2 years) in the one-jaw group and 27.7 years $(\mathrm{SD}=8.6$ years) in the two-jaw group.

\section{Thermography equipment}

Facial thermograms were taken with an emissivity of 0.98 using a Thermo Shot F30 camera (NEC Avio Infrared Technologies Co. Ltd., Tokyo, Japan), which had a thermal sensitivity of 0.1 degree and an accuracy of $+/-2 \%$. Non-uniformity correction included in the camera automatically adjusted the intrinsic reproducibility to less than $1 \%$.

\section{Facial imaging}

Before taking the facial thermograms, each patient's face was cleared of hair, wiped with a damp cloth, then air-dried with a small electric fan. To allow equilibration of the facial temperature, each patient was seated in a room at $22^{\circ} \mathrm{C}$ for $15 \mathrm{~min}$. Each patient's right profile was then cooled for $30 \mathrm{~s}$ using a refrigerant kept at $0^{\circ} \mathrm{C}$. 
This cooling protocol was adopted following the suggestions by Symonds et al. (19) and Ang et al. (20), that a cooling challenge was required to evaluate changes in a local temperature within a limited time. In their studies, feet or hands were cooled with water at $18^{\circ} \mathrm{C}(20)$ and $20^{\circ} \mathrm{C}$ (19) for $5 \mathrm{~min}$. For patients who have undergone orthognathic surgery and intermaxillary fixation for 7 days, it is difficult to retain the same head position for prolonged periods for sequential thermography. Therefore, to shorten the thermography time, the refrigerant was stored and cooled in a freezer, kept at $0^{\circ} \mathrm{C}$, and then applied to the right profile for $30 \mathrm{~s}$. The camera was placed on a tripod facing the patient's right side, aligned with the patient's cheek, and positioned with the lens at a $90^{\circ}$ angle, $50 \mathrm{~cm}$ from the patient's face. The lens was then focused on the patient's cheek.

A sequence of standardized thermograms of the patient's right cheek was taken using the thermographic camera 1 day prior to orthognathic surgery (T1) and at release from intermaxillary fixation (T2). Thermogram sequences were also taken at discharge from hospital. However, six of the ten patients in the two-jaw group dropped out, making the sample size too small for statistical comparisons. As a result, thermograms taken at discharge from hospital were not included in this study. All images were stored on computer-readable storage media for facial temperature analysis. The thermography sequence was seven images taken over a period of $3 \mathrm{~min}$ at $30-\mathrm{s}$ intervals.

\section{Image analysis}

Of the seven facial thermograms taken at $\mathrm{T} 1$ and $\mathrm{T} 2$ for each patient, the two taken $30 \mathrm{~s}$ (TG1) and $3 \mathrm{~min}$ (TG2) after the start of recording were used in this study. Selection of two thermograms (TG1, TG2) was adopted based on the statement by Ang et al. (20) that processing of thermogram sequences is time-consuming, longer sequences requiring longer processing times. To prevent any measurement bias, the thermograms for each patient were coded and numbered on a computer by one of the co-authors (AK). For each patient, each of the four facial thermograms was calibrated by superimposition of the orbitale and the tragion on the orbitale and the porion of the lateral cephalogram, respectively, which was taken at $\mathrm{T} 1$.

A square with four equal sides 26 pixels long was marked on each thermogram using the software provided by the camera manufacturer. The location of the square was decided by superimposing one vertex and one side of the square on the orbitale and the Frankfort horizontal plane, respectively, as shown in Fig. 1. The mean facial

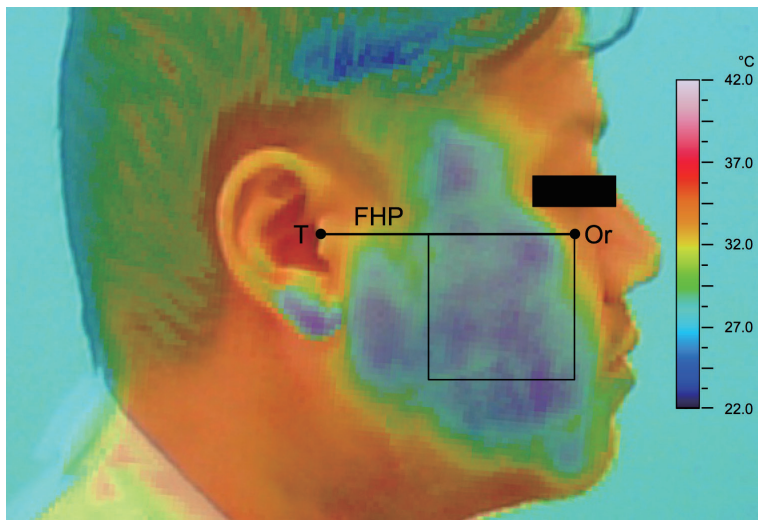

Fig. 1 Measurement of facial temperature for the square with four 26-pixels-long sides. Or: orbitale; T: tragion; FHP: Frankfort horizontal plane.

temperature for this square area was measured on TG1 and TG2 at T1 and T2 in each patient. A single investigator (KK) assessed all of the thermograms.

\section{Statistical analysis}

BellCurve for Excel (version 2.15, Social Survey Research Information, Tokyo, Japan) was used for statistical analyses. Three-way repeated-measures ANOVA was used to analyze the effects of the group (betweensubject factor, two levels, the one- and two-jaw groups), the time (within-subject factor, two levels, T1 and T2), and the thermogram (within-subject factor, two levels, TG1 and TG2) on facial temperature after testing the normality of the distribution and homogeneity of the variance. The level of significance was set at $5 \%$.

\section{Measurement error}

For evaluation of measurement errors, 20 randomly selected thermograms were measured again by the same investigator (KK) 1 month later. Measurement error, calculated according to Dahlberg's formula (21), was $0.32^{\circ} \mathrm{C}$, which was unlikely to affect the significance of the results obtained. Paired $t$ tests with a 95\% confidence interval revealed no systematic error.

\section{Results}

Table 1 shows the data for facial temperature on each thermogram at each time point in each group.

Shapiro Wilk and Leven tests confirmed the normality of the distribution and the homogeneity of the variance for facial temperature, respectively. Therefore, 3-way repeated measures ANOVA was performed for Table 1, and the results are shown in Table 2 , group $\times$ time, group $\times$ thermogram, or group $\times$ time $\times$ thermogram interactions having no significant effects on facial temperature. This table also demonstrates that the group, the time and the 
Table 1 Descriptive statistics of facial temperature $\left({ }^{\circ} \mathrm{C}\right)$

\begin{tabular}{|c|c|c|c|c|c|c|c|c|}
\hline & \multicolumn{4}{|c|}{ One-jaw group } & \multicolumn{4}{|c|}{ Two-jaw group } \\
\hline & \multicolumn{2}{|c|}{$\mathrm{T} 1$} & \multicolumn{2}{|c|}{$\mathrm{T} 2$} & \multicolumn{2}{|c|}{$\mathrm{T} 1$} & \multicolumn{2}{|c|}{$\mathrm{T} 2$} \\
\hline & Mean & SD & Mean & $\mathrm{SD}$ & Mean & $\mathrm{SD}$ & Mean & $\mathrm{SD}$ \\
\hline TG1 & 27.12 & 1.10 & 28.22 & 2.58 & 27.87 & 1.97 & 30.78 & 1.52 \\
\hline TG2 & 30.97 & 1.31 & 31.51 & 1.30 & 31.73 & 1.34 & 33.59 & 0.99 \\
\hline
\end{tabular}

SD indicates standard deviation.

Table 2 Results of three-way analysis of variance for factors evaluated

\begin{tabular}{llrrrrr}
\hline & & Sum of squares & df & Mean square & F & Significance \\
\hline Between subjects & Group & 36.978 & 1 & 36.978 & 7.470 & $<0.05$ \\
& Error & 89.110 & 18 & 4.951 & & \\
Within subjects & 53.677 & 1 & 53.677 & 13.263 & $<0.05$ \\
& Time & 13.423 & 1 & 13.423 & 3.32 & NS \\
& Group $\times$ Time & 72.847 & 18 & 4.047 & & \\
& Error (Time) & 254.220 & 1 & 254.220 & 292.123 & $<0.001$ \\
& Thermogram & 0.002 & 1 & 0.002 & 0.002 & NS \\
Group $\times$ Thermogram & 15.665 & 18 & 0.870 & & \\
Error (Thermogram) & 3.859 & 1 & 3.859 & 4.673 & $<0.05$ \\
& Time $\times$ Thermogram & 0.533 & 1 & 0.533 & 0.6455 & NS \\
Group $\times$ Time $\times$ Thermogram & Error (Time $\times$ Thermogram) & 14.864 & 18 & 0.826 & & \\
\hline
\end{tabular}

Table 3 Effects of factors on facial temperature $\left({ }^{\circ} \mathrm{C}\right)$

\begin{tabular}{|c|c|c|c|c|}
\hline \multirow[b]{2}{*}{ Factor } & & \multirow[b]{2}{*}{$n$} & \multicolumn{2}{|c|}{ Facial temperature } \\
\hline & & & Mean & $\mathrm{SD}$ \\
\hline \multirow[t]{2}{*}{ Group } & One jaw group & 40 & 29.46 & 2.46 \\
\hline & Two jaw group & 40 & 30.82 & 2.69 \\
\hline \multirow[t]{2}{*}{ Time } & $\mathrm{T} 1$ & 40 & 29.32 & 2.48 \\
\hline & $\mathrm{T} 2$ & 40 & 30.96 & 2.59 \\
\hline \multirow[t]{2}{*}{ Thermogram } & TG1 & 40 & 28.35 & 2.31 \\
\hline & TG2 & 40 & 31.92 & 1.55 \\
\hline
\end{tabular}

thermogram had significant effects on facial temperature.

Facial temperatures were significantly higher in the two-jaw group at T2 and on TG2 than in the one-jaw group at $\mathrm{T} 1$ and on TG1, respectively (Tables 2 and 3).

For facial temperature, three-way ANOVA confirmed the significant time $\times$ thermogram interaction (Table 2). Simple main effects showed that facial temperatures were significantly higher at $\mathrm{T} 2$ than at $\mathrm{T} 1$ on both TG1 and TG2, and were significantly higher on TG2 than on TG1 at both T1 and T2 (Table 4, Fig. 2).

\section{Discussion}

The null hypothesis, that orthognathic surgery and intermaxillary fixation have no effect on facial temperature, was rejected. The mean temperature differences between the levels of the factors, which showed a significant difference and a significant interaction, were $1.36^{\circ} \mathrm{C}$ for the group (Table 3), more than $1.2^{\circ} \mathrm{C}$ for the time, and more than $3.13^{\circ} \mathrm{C}$ for the thermogram (Table 4). These values were larger than the mean temperature differences found in previous studies, thus confirming that thermography was useful for assessing chronic orofacial pain disorders (10), temporomandibular joint disorders $(6,11)$, inferior alveolar nerve injury after orthognathic surgery (12), and inflammation after mandibular third molar extraction (13-15). Moreover, these mean temperature differences were larger than the random error $\left(0.32^{\circ} \mathrm{C}\right)$, thus demonstrating that thermography had high reproducibility for measurement of facial temperature. Therefore, thermography was considered useful for quantitative assessment of facial temperature in patients undergoing orthognathic surgery. This is in agreement with Christensen et al. (16), who showed that thermography was sufficiently sensitive for detection of inflammation after mandibular third molar extraction, and might be useful for assessing inflammation after more extensive surgical procedures such as orthognathic surgery.

Wall et al. (22) have found that muscle inactivity or 
Table 4 Facial temperature $\left({ }^{\circ} \mathrm{C}\right)$ according to a significant time $\times$ thermogram interaction

\begin{tabular}{lccccc}
\hline & \multicolumn{9}{c}{ Time } & \\
\cline { 2 - 5 } Thermogram & \multicolumn{2}{c}{ T1 $(n=20)$} & T2 $(n=20)$ & Simple main effect \\
\cline { 2 - 5 } TG1 $(n=20)$ & 27.31 & 1.55 & 29.39 & 2.50 & $P<0.001$ \\
TG2 $(n=20)$ & 31.32 & 1.34 & 32.52 & 1.53 & $P<0.05$ \\
Simple main effect & \multicolumn{2}{c}{$P<0.001$} & \multicolumn{2}{c}{$P<0.001$} & \\
\hline
\end{tabular}

$n$ indicates the number of thermograms; SD: standard deviation.

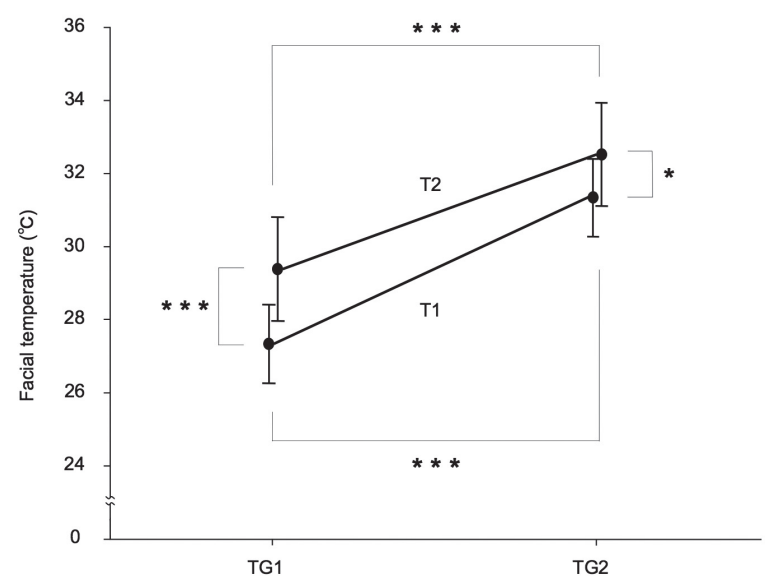

Fig. 2 Statistical analyses of facial temperature $\left({ }^{\circ} \mathrm{C}\right)$ according to a significant time $\times$ thermogram interaction. $* P<0.05, * * * P<0.001$.

disuse leads to sarcopenia, characterized by rapid loss of skeletal muscle. Geiser et al. (23) reported that disuse atrophy was characterized by substantially reduced body temperature. These studies suggested that muscle atrophy during intermaxillary fixation led to a decrease in facial temperature. It might be expected that facial temperature would be increased by inflammation after orthognathic surgery and decreased by sarcopenia of masticatory muscles resulting from intermaxillary fixation. In the present study, thermograms taken at $30 \mathrm{~s}$ (TG1) and 3 minutes (TG2) after the start of recording showed that facial temperatures were significantly higher after orthognathic surgery and at release from intermaxillary fixation (T2) than before these procedures (T1) (Table 4, Fig. 2). These results suggested that orthognathic surgery and intermaxillary fixation inhibited the decrease in facial temperature upon application of refrigerant, and promotion of facial temperature recovery after cooling. It can be speculated that these changes in facial temperature were due predominantly to inflammation after orthognathic surgery, rather than to sarcopenia. This is supported by the findings of Biagioni et al. (13), Venta et al. (14), Lee et al. (12), and Christensen et al. (15). After extraction of mandibular third molars, Biagioni et al. (13), Venta et al. (14), and Christensen et al. (15) showed that facial temperature was significantly higher on the operation side than on the control side. Lee et al. (12) reported that in patients undergoing orthognathic surgery, the skin supplied by the damaged inferior alveolar nerve after surgery became warm usually for a few months.

The thermograms taken at $3 \mathrm{~min}$ after the start of recording (TG2) demonstrated significantly higher facial temperatures than those taken at $30 \mathrm{~s}$ after the start of recording (TG1) before (T1) and after (T2) orthognathic surgery and release from intermaxillary fixation (Table 4). These results were expected, given that removal of the refrigerant from the right profile would lead to normalization of facial temperature to the original level.

In this study, facial temperature was higher in the two-jaw group than in the one-jaw group (Table 3). One possible reason for the significant inter-group difference in facial temperature might have been because the two-jaw surgery patients who underwent both Le Fort I osteotomy and bilateral SSRO had a wider range of inflammation than the one-jaw surgery patients who had undergone bilateral SSRO alone. This appears to be supported by van der Vlis et al. (24), who reported that, in the first 3 postoperative weeks, facial swelling was greater after bimaxillary surgery than after single surgery. Another possible reason might be the difference in the amount of jaw displacement. This appears to be supported by the present $t$ test, which showed that the total displacement of the mandible and the maxilla in the two-jaw group $(11.3 \mathrm{~mm})$ was significantly larger than the amount of mandibular set-back in the one-jaw group $(5.2 \mathrm{~mm})$. Facial temperature changes can thus be affected by the degrees of jaw set-back and set-forward.

It has been reported that women have slightly higher temperatures than men, and that older individuals have significantly lower $\left(0.5^{\circ} \mathrm{C}\right)$ temperatures $(25)$. One limitation of this study was that it was impossible to analyze the effect of gender on facial temperature because of the inadequate sample size and the improper gender distribution of the groups, although analysis using three-way repeated-measures ANOVA showed sufficient power of more than 0.8 .

The conclusions of this study can be briefly summa- 
rized as follows: 1) Thermography was useful for quantitative assessment of facial temperature in patients undergoing orthognathic surgery; and 2) Changes in facial temperature were due predominantly to inflammation after orthognathic surgery, rather than to sarcopenia.

\section{Acknowledgement}

This study was supported by a Grant-in Aid for Scientific Research (C) from the Japan Society for the Promotion of Science (Grant Number 15K11374).

\section{Conflict of interest}

The authors have no conflict of interest to declare.

\section{References}

1. Soh CL, Narayanan V (2013) Quality of life assessment in patients with dentofacial deformity undergoing orthognathic surgery-a systematic review. Int J Oral Maxillofac Surg 42, 974-980.

2. Son S, Kim SS, Son WS, Kim YI, Kim YD, Shin SH (2015) Miniscrews versus surgical archwires for intermaxillary fixation in adults after orthognathic surgery. Korean J Orthod 45, 3-12.

3. Bell WH, Gonyea W, Finn RA, Storum KA, Johnston C, Throckmorton GS (1983) Muscular rehabilitation after orthognathic surgery. Oral Surg Oral Med Oral Pathol 56, 229-235.

4. Hayter JP, Cawood JI (1993) The functional case for miniplates in maxillofacial surgery. Int J Oral Maxillofac Surg 22, 91-96.

5. Walston JD (2012) Sarcopenia in older adults. Curr Opin Rheumatol 24, 623-627.

6. Woźniak K, Szyszka-Sommerfeld L, Trybek G, Piątkowska D (2015) Assessment of the sensitivity, specificity, and accuracy of thermography in identifying patients with TMD. Med Sci Monit 21, 1485-1493.

7. Kim SJ, Yoo J, Kim YS, Shin SW (2010) Temperature change in pig rib bone during implant site preparation by low-speed drilling. J Appl Oral Sci 18, 522-527.

8. Kabbach W, Zezell DM, Pereira TM, Albero FG, Clavijo VR, de Andrade MF (2008) A thermal investigation of dental bleaching in vitro. Photomed Laser Surg 26, 489-493.

9. Iijima M, Yasuda Y, Muguruma T, Mizoguchi I (2010) Effects of $\mathrm{CO}_{2}$ laser debonding of a ceramic bracket on the mechanical properties of enamel. Angle Orthod 80, 1029-1035.

10. Gratt BM, Graff-Radford SB, Shetty V, Solberg WK, Sickles EA (1996) A 6-year clinical assessment of electronic facial thermography. Dentomaxillofac Radiol 25, 247-255.

11. McBeth SB, Gratt BM (1996) Thermographic assessment of temporomandibular disorders symptomology during orthodontic treatment. Am J Orthod Dentfacial Orthop 109, 481-488.

12. Lee JG, Kim SG, Lim KJ, Choi KC (2007) Thermographic assessment of inferior alveolar nerve injury in patients with dentofacial deformity. J Oral Maxillofac Surg 65, 74-78.

13. Biagioni PA, McGimpsey JG, Lamey PJ (1996) Electronic infrared thermography as a dental research technique. $\mathrm{Br}$ Dent J 180, 226-230.

14. Venta I, Hyrkas T, Paakkari I, Ylipaavalniemi P (2001) Thermographic imaging of postoperative inflammation modified by anti-Inflammatory pretreatment. J Oral Maxillofac Surg 59, 145-148.

15. Christensen J, Matzen LH, Vaeth M, Schou S, Wenzel A (2012) Thermography as a quantitative imaging method for assessing postoperative inflammation. Dentomaxillofacial Radiology 41, 494-499.

16. Christensen J, Matzen LH, Schou S, Væth M, Wenzel A (2014) Is thermography useful for assessment of postoperative inflammation after surgical removal of mandibular third molars when methylprednisolone is administered and how does it correlate with patients' perception of swelling? J Oral Maxillofac Surg 72, 463-469.

17. Boehmer TK, Patnaik JL, Burnite SJ, Ghosh TS, Gershman K, Vogt RL (2011) Use of hospital discharge data to evaluate notifiable disease reporting to Colorado's electronic disease reporting system. Public Health Rep 126, 100-106.

18. Faul F, Erdfelder E, Lang AL, Buchner A (2007) G*Power 3: a flexible statistical power analysis program for the social, behavioral, and biomedical sciences. Behavior Research Methods 39, 175-191.

19. Symonds ME, Henderson K, Elvidge L, Bosman C, Sharkey D, Perkins AC et al. (2012) Thermal imaging to assess agerelated changes of skin temperature within the supraclavicular region co-locating with brown adipose tissue in healthy children. J Pediatr 161, 892-898.

20. Ang QY, Goh HJ, Cao Y, Li Y, Chan SP, Swain JL et al. (2017) A new method of infrared thermography for quantification of brown adipose tissue activation in healthy adults (TACTICAL): a randomized trial. J Physiol Sci 67, 395-406.

21. Kim HY (2013) Statistical notes for clinical researchers: evaluation of measurement error 2: Dahlberg's error, Bland-Altman method, and kappa coefficient. Restor Dent Endod 38, 182-185.

22. Wall BT, Dirks ML, van Loon LJ (2013) Skeletal muscle atrophy during short-term disuse: implications for age-related sarcopenia. Ageing Res Rev 12, 898-906.

23. Geiser F, Currie SE, O'Shea KA, Hiebert SM (2014) Torpor and hypothermia: reversed hysteresis of metabolic rate and body temperature. Am J Physiol Regul Integr Comp Physiol 307, R1324-R1329.

24. van der Vlis $M$, Dentino KM, Vervloet $B$, Padwa BL (2014) Postoperative swelling after orthognathic surgery: a prospective volumetric analysis. J Oral Maxillofac Surg 72, 2241-2247.

25. Kelly G (2006) Body temperature variability (Part 1): a review of the history of body temperature and its variability due to site selection, biological rhythms, fitness, and aging. Altern Med Rev 11, 278-293. 